\title{
Evaluación de potencialidades en comunicación científica de universidades mexicanas
}

\author{
JaVier TARANGo \\ Perla Zukey Hernández-Gutiérrez \\ Profesores investigadores \\ Universidad Autónoma de Chihuahua
}

\section{Resumen}

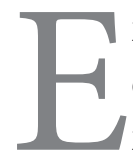

n este artículo se analizan los principales criterios para evaluar los niveles de divulgación científica en universidades mexicanas, se trata de un estudio comparativo entre las 10 principales universidades mexicanas caracterizadas por sus niveles de contribución a la generación y divulgación de conocimiento, en comparación con el comportamiento observado en las instituciones de educación superior del Estado de Chihuahua inmersas en este proceso.

Palabras clave: divulgación científica, producción académica, comunicación científica, potencialidades científicas, universidades mexicanas
Introducción

Existe un considerable volumen de publicaciones dedicadas a la identificación de literatura especializada sobre produccióncomunicación científica, así como las frecuencias y cantidades de productos generados. Sin embargo, muchos de estos trabajos han mostrado solo aspectos parciales y en ocasiones han estado limitados al análisis de una fuente, institución o indicador en particular; otros han abordado este tipo de estudios de manera más integral, mostrando tendencias en el comportamiento sobre la generación de objetos de divulgación en sistemas más amplios (estudios estatales, nacionales e internacionales), aunque de manera frecuente sin posibilidad de determinar el factor de impacto que tal producción científica fue capaz de propiciar. 
El registro de productos generados, representados únicamente en regularidades o frecuencias en el desarrollo de procesos de divulgación, suele no tener validez y rigor científico suficiente desde la perspectiva de distintos organismos evaluadores, ya que cuando esto sucede, tienden a considerar la inclusión de algunos elementos que no garantizan calidad en sus contenidos $y$ al no ser incluidos en fuentes de información con rigor científico (como revistas indizadas) es imposible pasar del registro de frecuencias en productos generados a la determinación de elementos de calidad en los mismos.

Las inequidades por desarrollar estudios en producción-comunicación científica en los niveles de educación superior en México, tanto en instituciones públicas como privadas, cobra relevancia para la identificación de elementos comparativos entre países, disciplinas e instituciones, tomando como referencia aquellos elementos de medición que se apegan a los estudios fundamentados en indicadores métricos de información (que evalúan la calidad por encima de la cantidad de los productos) y las normatividades internacionales (que reconocen fuentes, productos y condiciones de divulgación específicas).

Por tanto, la identificación de producción-comunicación científica no debe incluir en sus registros cualquier forma de divulgación impresa o electrónica sin considerar la evaluación previa hecha por instancias de arbitraje, quienes determinaron sus condiciones de calidad y reconocimiento ante estándares internacionales. Por ejemplo, en el estado de Chihuahua se ha realizado un esfuerzo por registrar la producción científica que incluye publicaciones y ponencias presentadas en congresos nacionales y locales (Martínez Escárcega y Vega Villarreal, 2012), sin embargo, los resultados que se proporcionan tienen poco que ver con la visión generada por organismos nacionales e internacionales como el Consejo Nacional de Ciencia y Tecnología (CONACYT) o el Institute for Scientific Information (ISI) de Pensilvania, Philadelphia, Estados Unidos, además de múltiples autores (Mondrego, 2002; Maletta, 2009 y González Nando, 2007), quienes ponderan al artículo científico publicado en revista arbitrada e indizada por encima del libro, capítulo de libro, tesis y ponencia, siendo esta fuente la única en la que se puede medir el verdadero impacto del conocimiento de forma sistemática.

Los esfuerzos por desarrollar registros sistemáticos sobre divulgación de conocimiento a nivel estatal son francamente loables, convirtiéndose en observatorios científicos de la entidad, sin embargo, se hace necesario sobrepasar tales condiciones al integrar investigaciones relacionadas con la identificación de potencialidades institucionales o regionales que puedan ser evaluadas con parámetros de calidad, cuando menos a nivel nacional.

Este estudio es una aproximación a la identificación de condiciones institucionales en producción-comunicación científica, ampliando los elementos de estudio a otros actores más allá del mero objeto como medio de divulgación, por lo que integra a otros actores fundamentales como: instituciones de educación superior (públicas 
y privadas), docentes e investigadores (registrados como capital humano dentro de instancias evaluadoras), programas educativos y medios de divulgación arbitrados e indizados (abordados por separado).

Dada la disposición de información (los datos más recientes corresponden a 2010) y el propósito del estudio de identificar potencialidades entre una realidad local (estado de Chihuahua) contra una realidad nacional (México); el estudio analiza las condiciones de producción-comunicación científica de las 10 universidades más preponderantes en el país en generación y divulgación del conocimiento, para luego contextualizar la realidad estatal de las universidades públicas y privadas, lo que propicia el reconocimiento de elementos para la determinación de potencialidades locales en relación con las condiciones identificadas a nivel nacional.

\section{Conceptualización de la producción/ comunicación científica}

El concepto de producción puede ser asociado con múltiples actividades, principalmente de carácter industrial, pero poco asociado con el desarrollo de la ciencia. Dentro de los ámbitos de investigación científica suelen usarse en diferentes tiempos y momentos varios conceptos relacionados con esto: producción científica, producción académica y comunicación científica, regularmente los dos primeros se manejan como sinónimos y el tercero se ubica como una consecuencia de los anteriores.

La identificación de los insumos que requieren los investigadores, así como su transformación, suelen ser definidos por los involucrados en procesos investigativos. La mayor dificultad estriba en una clasificación que sea reconocida como producto de la actividad sistemática y organizada, plasmada en escritos y otras formas de divulgación válidas, siempre y cuando cumplan con los niveles de calidad establecidos.

Los productos que comprende la comunicación científica pueden incluir diversos tipos, entre los más comunes están los libros, capítulos de libros, tesis, proyectos de investigación (Braga, Bernadete y Silva, 2003), por mencionar los principales, sin embargo, un producto clave es el artículo científico. Luna y Russell (2009), consideran que la finalidad de este tipo de documento es comunicar resultados de investigación, ideas y debates de manera clara, concisa y fidedigna, siendo el proceso que conduce hacia los últimos resultados de investigación.

La labor de reflexión teórica, así como la búsqueda y análisis de datos empíricos, cuyos resultados luego se han de volcar en productos escritos, es una labor de investigación científica (teórica o empírica, usualmente ambas a la vez). La labor de escribir informes, artículos o libros, en los que se vuelca la creación del conocimiento científico obtenido mediante la investigación, es una labor de comunicación científica o comunicación académica. El conjunto de las dos constituye un proceso indivisible que se puede denominar producción científica o producción académica. La fase de comunicación es esencial en este proceso, porque permite validar los resultados científicos sometiéndolos a la discusión y al juicio de la comunidad profesional (Maletta, 2009). 
Al respecto, Romanos de Tiratel (2009) indica que, la comunicación científica es un proceso mediante el cual interactúan los científicos creando nuevo conocimiento. La difusión de dichas investigaciones se hace por medio de canales informales, semiformales o formales (Campos, 2000), estos últimos son los únicos viables de ser considerados como productos que proporcionan información suficiente para desarrollar metrías de información en una determinada disciplina.

Los nuevos modelos de comunicación científica muestran que ya casi desaparece el contacto cara a cara en seminarios, coloquios y congresos, surgiendo como consecuencia medios de divulgación basados en tecnología conocidos como servidores o repositorios electrónicos, en donde los investigadores discuten y argumentan, comparten y buscan información, escriben y eligen el formato más adecuado para comunicarse.

En la actualidad se debate acerca de si la comunicación científica se está transformando por el uso de las tecnologías de la información y comunicación (TIC) desde el proceso de investigación, hasta la difusión y el consumo.

El cálculo de la producción científica o académica de los docentes universitarios en general, ha estado fuertemente relacionado con su compromiso dentro de la propia institución. La producción académica se tasa por el número de publicaciones que un investigador ha desarrollado en determinado tiempo, esto puede ser comprobado por medio del acceso a distintos mecanismos formales de concentración de in- formación de esta naturaleza, que evalúan la calidad de los productos, sin necesidad de recurrir directamente al investigador.

La producción científica tiene dos partes esenciales que son: la investigación y la comunicación de los hallazgos. Por lo tanto la aplicación del conocimiento es necesaria para crear la producción científica. Para Mondrego (2002), la producción científica o conjunto de resultados alcanzados, se convierte en una continua espiral de conocimientos y otros recursos puestos en acción, los cuales al incorporarse a la sociedad configuran el matiz de la cultura de la ciencia.

El trabajo de reflexión teórica, búsqueda y análisis de datos, que se convierten en productos escritos forman parte de la labor de la investigación científica. La comunicación científica es la que permite validar los resultados, ya que son expuestos a discusión y al juicio de la comunidad científica correspondiente. Si no se da la comunicación científica no hay ciencia. Aunque algunos autores han abogado por la inclusión de indicadores de productividad de investigación como el número de patentes, presentaciones en conferencias y libros de texto publicados, que no se limitan al conteo de artículos en revistas indexadas o a la publicación (García-Cepero, 2010).

\section{Materiales y método}

Dado que la comparación en la evaluación de potencialidades de las instituciones de educación superior (IES) mexicanas, públicas y privadas, requiere de la búsqueda de aquellos elementos que proporcionen conocimiento para mejorar los 
resultados, el método que se utiliza en este tipo de estudios se basa en la investigación documental recurriendo a fuentes especializadas, dedicadas de forma oficial al procesamiento de los datos pertinentes en relación con la producción científica de las instituciones que la desarrollan.

Para este fin, se trabajó con:

1) Un estudio comparativo de universidades mexicanas creado por la Dirección de Evaluación Institucional de la UnAM (2010) que concentra los resultados de 2,626 instituciones de educación superior (públicas y privadas) por tipo, por conjuntos institucionales y por sector, correlacionando variables por entidades federativas. Por la dimensión de la información acumulada, se consideraron únicamente a las universidades públicas y privadas que se ubicaran en cada indicador dentro de las 10 de mayor rango, presentando posteriormente los resultados de IEs estatales que ingresaron dentro del rango de las primeras 60 , resultando en dos casos únicamente la Universidad Autónoma de Chihuahua (UACH) y la Universidad Autónoma de Ciudad Juárez (UACJ) para el caso de todos los indicadores. Tales datos corresponden al año 2010.

2) Información proporcionada por el Conacyt (2012) en relación con la distribución de miembros vigentes del Sistema Nacional de Investigadores (SNI), con especial énfasis en aquellos pertenecientes a instituciones diversas ubicadas en el Estado de Chihuahua.
La identificación de indicadores que determinaron la realidad y potencialidad fueron: matrícula de estudiantes, docentesinvestigadores, miembros pertenecientes a SNI, evaluación de programas educativos, publicaciones registradas en ISI (artículos indizados), SCOPUS, CLASE y PERIODICA (artículos arbitrados y de divulgación), generación de patentes y generación de publicaciones para divulgación del conocimiento.

\section{Análisis de resultados}

El análisis parte de identificar la matrícula y la proporción de alumnos por docentes como el núcleo principal de una universidad, ya que de ello dependen los recursos y actividades que se desarrollan en las IES. La tabla 1 relaciona las 10 universidades mexicanas (ocho públicas y dos privadas) con mayor población estudiantil y las IES estatales, y la proporción alumno-maestro: la UNAM tiene la proporción más baja y la Universidad Pedagógica Nacional (analizada como sistema nacional y no como unidades independientes) la más alta (7.29 y 13.58 alumnos promedio por docente).

Este indicador resulta especialmente revelador ya que a menor número de estudiantes atendidos puede inferirse que los profesores disponen de mayor tiempo para actividades de investigación y por lo tanto, de comunicación científica. Tanto la UACH como la UACJ como instituciones locales se encuentran por encima de la media en este indicador. 
REVISTA DE INVESTIGACIÓN EDUCATIVA DE LA REDIECH N. 5

Tabla 1. Promedio de alumnos atendidos por docentes

\begin{tabular}{|l|l|l|l|l|}
\hline $\begin{array}{l}\text { Posición } \\
\text { respecto } \\
\text { al criterio }\end{array}$ & IES & $\begin{array}{l}\text { No. Matri- } \\
\text { cula }\end{array}$ & $\begin{array}{l}\text { No. Docen- } \\
\text { tes }\end{array}$ & $\begin{array}{l}\text { Promedio } \\
\text { alumnos por } \\
\text { docente }\end{array}$ \\
\hline 1 & Universidad Nacional Autónoma de México & 199535 & 27361 & 7.29 \\
\hline 2 & Instituto Politécnico Nacional & 93525 & 9938 & 9.41 \\
\hline 3 & Universidad de Guadalajara & 86792 & 7571 & 11.46 \\
\hline 4 & Universidad Autónoma de Nuevo León & 75809 & 5820 & 13.03 \\
\hline 5 & Sistema Tecnológico de Monterrey & 70771 & 7665 & 9.23 \\
\hline 6 & Universidad Pedagógica Nacional & 67673 & 4985 & 13.58 \\
\hline 7 & Sistema Universidad Valle de México & 66044 & 8917 & 7.41 \\
\hline 8 & Universidad Autónoma Metropolitana & 50404 & 5809 & 8.68 \\
\hline 9 & Universidad Autónoma de Baja California & 38197 & 4535 & 8.42 \\
\hline 10 & Universidad Autónoma del Estado de México & 38197 & 4875 & 7.84 \\
\hline 20 & Universidad Autónoma de Chihuahua & 25099 & 2400 & 10.46 \\
\hline 24 & Universidad Autónoma de Ciudad Juárez & 21116 & 2089 & 10.10 \\
\hline
\end{tabular}

El primer indicador formal de identificación del proceso de comunicación científica, es el número de docentes que son miembros del Sistema Nacional de Investigadores (SIN) por IES. Este organismo es el máximo órgano en promover el desarrollo de las actividades concernientes a la investigación. Tanto para ser admitido como para permanecer en el SNI se consideran las aportaciones al conocimiento científico, tecnológico, social y cultural, mediante la investigación científica y/o tecnológica, la divulgación de la ciencia, la formación de grupos de investigación, el desarrollo de infraestructura científica y tecnológica, así como las labores para vincular la actividad de investigación con los sectores públicos, social y privado (SNI, 2012).
En la tabla 2 se muestra la relación de las 10 IES con mayor población de docentes, seguida por el número de miembros en el SNI, de lo cual se deriva el porcentaje de docentes que gozan de tal reconocimiento en relación con la población total institucional, en donde la Universidad Autónoma Metropolitana (UAM) tiene el mejor indicador (15.60\%) seguida por la UNAM con $13.10 \%$ y la de menor rango se ubica en el Sistema Universidad Valle de México (0.07\%), de carácter privado, a pesar de su preponderante población y distribución territorial en el país. En el caso de las universidades estatales que cuentan con miembros en SNI, se observan indicadores bajos ubicándolas en el lugar 27 y 39 de la lista general. 
Tabla 2. Proporción de docentes pertenecientes al SNI

\begin{tabular}{|c|l|c|c|c|}
\hline $\begin{array}{l}\text { Posición } \\
\text { respecto } \\
\text { al criterio }\end{array}$ & \multicolumn{1}{|c|}{ IES } & No. Docentes & $\begin{array}{c}\text { Miembros } \\
\text { SNI }\end{array}$ & $\begin{array}{c}\text { \% de docentes } \\
\text { en SNI }\end{array}$ \\
\hline 1 & Universidad Nacional Autónoma de México & 27361 & 3583 & 13.10 \\
\hline 2 & Instituto Politécnico Nacional & 9938 & 779 & 7.84 \\
\hline 3 & Universidad de Guadalajara & 7571 & 662 & 8.74 \\
\hline 4 & Universidad Autónoma de Nuevo León & 5820 & 443 & 7.61 \\
\hline 5 & Sistema Tecnológico de Monterrey & 7665 & 264 & 3.44 \\
\hline 6 & Universidad Pedagógica Nacional & 4985 & 264 & 5.30 \\
\hline 7 & Sistema Universidad Valle de México & 8917 & 6 & 0.07 \\
\hline 8 & Universidad Autónoma Metropolitana & 5809 & 906 & 15.60 \\
\hline 9 & Universidad Autónoma de Baja California & 4535 & 216 & 4.76 \\
\hline 10 & Universidad Autónoma del Estado de México & 4875 & 288 & 5.91 \\
\hline 27 & Universidad Autónoma de Ciudad Juárez & 2400 & 97 & 0.04 \\
\hline 39 & Universidad Autónoma de Chihuahua & 2089 & 50 & 0.02 \\
\hline
\end{tabular}

Respecto a la comunicación científica, la mejor representación de arbitraje estricto y garantía de un producto publicado aparece en aquellas instituciones que pertenecen al ISI, empresa reconocida por los órganos evaluadores como el SNI y PROMEP para esta categoría de publicaciones, ya que es ahí donde se evalúa el factor de impacto de cada artículo científico publicado y ofrece servicios de bibliografía y análisis de citación a través del servicio Web of Science y estudios de factor de impacto y visibilidad a través del Journal Citation Report (JCR).
De acuerdo a los resultados arrojados en la tabla 3 respecto al promedio de artículos incluidos en ISI en relación con el número de miembros de SNI, se observa que el indicador más alto lo tiene el Sistema Universidad Valle de México quien con pocos miembros en SNI alcanza un promedio de 2.17 artículos por docente, sin embargo, la UNAM con un promedio de 0.81 supera en mucho al resto de las IES enlistadas en el número de artículos publicados. La UACH y la UACJ, a nivel local, se ubican en el lugar 39 y 44 respectivamente. 
REVISTA DE INVESTIGACIÓN EDUCATIVA DE LA REDIECH N. 5

Tabla 3. Artículos publicados por IES en ISI por miembros de SNI

\begin{tabular}{|c|l|c|c|c|}
\hline $\begin{array}{l}\text { Posición } \\
\text { respecto } \\
\text { al criterio }\end{array}$ & \multicolumn{1}{|c|}{ IES } & $\begin{array}{c}\text { Número de } \\
\text { miembros } \\
\text { SNI }\end{array}$ & $\begin{array}{c}\text { Artículos } \\
\text { en ISI }\end{array}$ & $\begin{array}{c}\text { Promedio de } \\
\text { artículos por } \\
\text { docente }\end{array}$ \\
\hline 1 & Universidad Nacional Autónoma de México & 3583 & 2917 & 0.81 \\
\hline 2 & Instituto Politécnico Nacional & 779 & 655 & 0.84 \\
\hline 3 & Universidad de Guadalajara & 662 & 283 & 0.43 \\
\hline 4 & Universidad Autónoma de Nuevo León & 443 & 288 & 0.65 \\
\hline 5 & Sistema Tecnológico de Monterrey & 264 & 144 & 0.55 \\
\hline 6 & Universidad Pedagógica Nacional & 264 & 0 & 0.00 \\
\hline 7 & Sistema Universidad Valle de México & 6 & 13 & 2.17 \\
\hline 8 & Universidad Autónoma Metropolitana & 906 & 526 & 0.58 \\
\hline 9 & Universidad Autónoma de Baja California & 216 & 146 & 0.68 \\
\hline 10 & Universidad Autónoma del Estado De México & 288 & 139 & 0.48 \\
\hline 39 & Universidad Autónoma de Chihuahua & 50 & 35 & 0.70 \\
\hline 44 & Universidad Autónoma de Ciudad Juárez & 97 & 19 & 0.19 \\
\hline
\end{tabular}

Otro medio de identificación de los niveles de comunicación científica de docentes en las IEs es Scopus, el cual se considera aglutina artículos que gozan de alta calidad, catalogados en el nivel de arbitrados. Este servicio, según Codina (2005) es una de las fuentes más importantes de difusión del conocimiento científico a través de publicaciones seriadas (revistas), visualiza la producción de investigadores en diversas regiones del planeta, difunde diferentes metodologías investigativas y genera redes académicas, proporciona acceso a 14,000 publicaciones de 4,000 editoriales con 27 millones de referencias. Dadas las caracte- rísticas del total de documentos incluidos en este tipo de servicios de información, se consideran artículos de difusión y divulgación.

La tabla 4 muestra las publicaciones, nuevamente de las 10 universidades con mayor población estudiantil y docente en relación con el número de artículos publicados en el navegador científico Scopus, en donde la UnAm ofrece el mejor índice con un total de 27361 docentes sólo el .10\% publica dentro de este navegador y de los profesores-investigadores miembros de SNI, únicamente el .78\%. 
Tabla 4. Artículos publicados por IES en SCOPUS por miembros de SNI

\begin{tabular}{|c|c|c|c|c|c|c|}
\hline $\begin{array}{l}\text { Posición } \\
\text { respecto } \\
\text { al criterio }\end{array}$ & IES & $\begin{array}{l}\text { No. Do- } \\
\text { centes }\end{array}$ & $\begin{array}{c}\text { Miem- } \\
\text { bros } \\
\text { SNI }\end{array}$ & $\begin{array}{c}\text { Artículos } \\
\text { en SCO- } \\
\text { PUS }\end{array}$ & $\begin{array}{l}\text { Promedio de } \\
\text { artículos en } \\
\text { SCOPUS por } \\
\text { docente }\end{array}$ & $\begin{array}{l}\text { Promedio de } \\
\text { artículos en } \\
\text { SCOPUS por } \\
\text { miembro SNI }\end{array}$ \\
\hline 1 & $\begin{array}{l}\text { Universidad Nacional Autónoma } \\
\text { de México }\end{array}$ & 27361 & 3583 & 2807 & 0.10 & 0.78 \\
\hline 2 & Instituto Politécnico Nacional & 9938 & 779 & 699 & 0.07 & 0.90 \\
\hline 3 & Universidad de Guadalajara & 7571 & 662 & 248 & 0.03 & 0.37 \\
\hline 4 & $\begin{array}{l}\text { Universidad Autónoma de Nuevo } \\
\text { León }\end{array}$ & 5820 & 443 & 277 & 0.05 & 0.63 \\
\hline 5 & Sistema Tecnológico de Monterrey & 7665 & 264 & 171 & 0.02 & 0.65 \\
\hline 6 & Universidad Pedagógica Nacional & 4985 & 264 & 3 & 0.00 & 0.01 \\
\hline 7 & $\begin{array}{l}\text { Sistema Universidad Valle de } \\
\text { México }\end{array}$ & 8917 & 6 & 7 & 0.00 & 1.17 \\
\hline 8 & $\begin{array}{l}\text { Universidad Autónoma Metropo- } \\
\text { litana }\end{array}$ & 5809 & 906 & 619 & 0.11 & 0.68 \\
\hline 9 & $\begin{array}{l}\text { Universidad Autónoma de Baja } \\
\text { California }\end{array}$ & 4535 & 216 & 144 & 0.03 & 0.67 \\
\hline 10 & $\begin{array}{l}\text { Universidad Autónoma del Estado } \\
\text { de México }\end{array}$ & 4875 & 288 & 183 & 0.04 & 0.64 \\
\hline 39 & $\begin{array}{l}\text { Universidad Autónoma de Chihu- } \\
\text { ahua }\end{array}$ & 2089 & 50 & 29 & 0.01 & 0.58 \\
\hline+60 & $\begin{array}{l}\text { Universidad Autónoma de Ciudad } \\
\text { Juárez }\end{array}$ & 2400 & 97 & 5 & 0.002 & 0.05 \\
\hline
\end{tabular}

A mitad de los años setenta, la UNAM creó las bases de datos Clase y Periódica. Estos servicios de información bibliográfica incluyen 400,000 artículos publicados en más de 2,800 revistas de 24 países de América Latina y el Caribe. La selección de las revistas debe hacerse considerando dos características principales: la multidisciplinariedad de los contenidos y el origen geográfico de las publicaciones. En este trabajo, se hace referencia al nuevo modelo de selección utilizado, el cual propone 30 criterios que indagan sobre la calidad de cada revista en tres ámbitos editoriales: a) la normalización editorial, b) la gestión y visibilidad de la revista, y c) la calidad de sus contenidos. Esta información tiene un $71 \%$ de materiales disponibles en español, un $18 \%$ en portugués y $11 \%$ en inglés.

En la tabla 5 se analiza el número de publicaciones en Clase y Periódica del total de docentes por IEs, en donde la UNAM cuenta con un total de 317 publicaciones y se puede corroborar que la UACH no publi- 
REVISTA DE INVESTIGACIÓN EDUCATIVA DE LA REDIECH N. 5

có en el año 2010 dentro de estos servi- $\quad$ y la UACJ, en el lugar 28, con una particicios de información ocupando el lugar 53 pación poco significativa.

Tabla 5. Artículos publicados por IES en CLASE y PERIÓDICA

\begin{tabular}{|c|l|c|}
\hline $\begin{array}{l}\text { Posición respecto } \\
\text { al criterio }\end{array}$ & \multicolumn{1}{|c|}{ IES } & CLASE Y PeriódicA \\
\hline 1 & Universidad Nacional Autónoma de México & 317 \\
\hline 2 & Instituto Politécnico Nacional & 25 \\
\hline 3 & Universidad de Guadalajara & 32 \\
\hline 4 & Universidad Autónoma de Nuevo León & 13 \\
\hline 5 & Sistema Tecnológico de Monterrey & 16 \\
\hline 6 & Universidad Pedagógica Nacional & 0 \\
\hline 7 & Sistema Universidad Valle de México & 3 \\
\hline 8 & Universidad Autónoma Metropolitana & 141 \\
\hline 9 & Universidad Autónoma de Baja California & 14 \\
\hline 10 & Universidad Autónoma del Estado de México & 46 \\
\hline 28 & Universidad Autónoma de Ciudad Juárez & 5 \\
\hline 53 & Universidad Autónoma de Chihuahua & 0 \\
\hline
\end{tabular}

Respecto a las patentes solicitadas $\mathrm{y}$ otorgadas (sector académico, serie 19912010), de acuerdo a la tabla 6, es posible comparar entre aquellas solicitadas contra las aceptadas. La IEs que mejor indicador mostró fue la UNAM con un $80.98 \%$ y la de menor rango dentro de las que presentaron propuestas para evaluación fue la Benemérita Universidad Autónoma de Puebla 12 solicitudes y ninguna aprobación. Las IES estatales incluidas en la lista (UACH y UACJ) presentan resultados nulos tanto en solicitud como en aprobación de patentes. 
Tabla 6. Comparación de patentes solicitadas y aceptadas por IES

\begin{tabular}{|c|l|c|c|c|}
\hline $\begin{array}{c}\text { Posición } \\
\text { respecto } \\
\text { al criterio }\end{array}$ & \multicolumn{1}{|c|}{ IES } & $\begin{array}{c}\text { Patentes } \\
\text { solicitadas }\end{array}$ & $\begin{array}{c}\text { Patentes } \\
\text { aceptadas }\end{array}$ & $\begin{array}{c}\text { \% de patentes } \\
\text { aceptadas }\end{array}$ \\
\hline 1 & Universidad Nacional Autónoma de México & 163 & 132 & 80.98 \\
\hline 2 & Sistema Tecnológico de Monterrey & 100 & 10 & 10.00 \\
\hline 3 & $\begin{array}{l}\text { Centro de Investigación y de Estudios Avan- } \\
\text { zados del IPN }\end{array}$ & 98 & 58 & 59.18 \\
\hline 4 & Universidad Autónoma Metropolitana & 90 & 59 & 65.56 \\
\hline 5 & Instituto Politécnico Nacional & 57 & 27 & 47.37 \\
\hline 6 & Universidad Autónoma de Nuevo León & 52 & 9 & 17.31 \\
\hline 7 & Universidad de Guanajuato & 33 & 7 & 21.21 \\
\hline 8 & Universidad de Guadalajara & 15 & 3 & 20.00 \\
\hline 9 & Universidad de Colima & 14 & 2 & 14.29 \\
\hline 10 & $\begin{array}{l}\text { Benemérita Universidad Autónoma de Pue- } \\
\text { bla }\end{array}$ & 12 & 0 & 0.00 \\
\hline 43 & Universidad Autónoma de Chihuahua & 0 & 0 & 0.00 \\
\hline 44 & Universidad Autónoma de Ciudad Juárez & 0 & 0 & 0.00 \\
\hline
\end{tabular}

En cuanto a la identificación de publicaciones por parte de las IEs como medios de divulgación del conocimiento generado por sus propios docentes e investigadores, así como de otras participaciones externas dentro del nivel de revistas arbitradas, el registro de dichas publicaciones en Latindex es una representación de tal indicador.

Latindex es un servicio de información fundado en 1995 por la UNAM como un servicio en red de cooperación regional en donde se divulgan las revistas de investigación científica, técnico profesionales y de divulgación científica y cultural que se editan en países de América Latina, El Caribe, España y Portugal. Latindex ofrece tres bases de datos: 1) directorio de datos biblio- gráficos y contactos de revistas registradas (impresas y electrónicas); 2) catálogo que incluye únicamente las revistas impresas o electrónicas que cumplen con los criterios de calidad editorial diseñados por Latindex; y 3) enlaces a revistas electrónicas que permiten el acceso a textos completos en los sitios que se encuentran disponibles (Latindex, 2012).

El número de revistas por IES siguiendo el modelo de ofrecer las 10 más representativas está en la tabla 7, en donde se observa que la Unam mantiene el liderazgo con 247 publicaciones registradas, ocupando la UACj y la UaCh las posiciones 36 y 41 con cuatro y tres publicaciones cada una respectivamente. 
REVISTA DE INVESTIGACIÓN EDUCATIVA DE LA REDIECH N. 5

Tabla 7. Número de revistas académicas en Latindex por IES

\begin{tabular}{|c|l|c|}
\hline Posición respecto al criterio & \multicolumn{1}{|c|}{ Universidad } & Revistas en LATINDEX \\
\hline 1 & Universidad Nacional Autónoma de México & 247 \\
\hline 2 & Universidad Autónoma Metropolitana & 70 \\
\hline 3 & Universidad de Guadalajara & 49 \\
\hline 4 & Universidad Veracruzana & 28 \\
\hline 5 & Benemérita Universidad Autónoma de Puebla & 26 \\
\hline 6 & Instituto Politécnico Nacional & 25 \\
\hline 7 & Sistema Universidad Iberoamericana & 24 \\
\hline 8 & Universidad Autónoma del Estado de México & 18 \\
\hline 9 & Universidad Autónoma de Nuevo León & 18 \\
\hline 10 & Universidad de Sonora & 18 \\
\hline 36 & Universidad Autónoma de Ciudad Juárez & 4 \\
\hline 41 & Universidad Autónoma de Chihuahua & 3 \\
\hline
\end{tabular}

Respecto a la generación de publicaciones indizadas, la instancia oficial para reconocer tal condición en México es el ConACYT, quien mantiene un índice con revistas científicas y tecnológicas como reconocimiento a su calidad y excelencia editorial. A través de un portal pone a disposición de la comunidad científica, la producción editorial a texto completo de las publicaciones incluidas en el índice mexicano de Investi- gación Científica y Tecnológica (Conacyt, 2012).

La divulgación del conocimiento a través de revistas incluidas en el índice de CoNACYT, el cual al 2010 se componía de 62 publicaciones emanadas de IES, de las cuales 29 son editadas por la UnAm. En el caso de la UACH y la UACJ no muestran ninguna publicación en esta categoría.

Tabla 8. Número de revistas en índice CONACYT

\begin{tabular}{|c|l|c|}
\hline Posición respecto al criterio & \multicolumn{1}{|c|}{ Universidad } & Revistas CoNACYT \\
\hline 1 & Universidad Nacional Autónoma de México & 29 \\
\hline 2 & Universidad Autónoma Metropolitana & 9 \\
\hline 3 & El Colegio de México & 7 \\
\hline 4 & Universidad Autónoma de Baja California & 4 \\
\hline 5 & Instituto Politécnico Nacional & 3 \\
\hline 6 & Sistema Tecnológico de Monterrey & 3 \\
\hline 7 & Universidad de Guadalajara & 3 \\
\hline 8 & Benemérita Universidad Autónoma de Puebla & 2 \\
\hline 9 & Universidad Autónoma Chapingo & 2 \\
\hline 10 & Universidad Autónoma del Estado de México & 2 \\
\hline 31 & Universidad Autónoma de Chihuahua & 0 \\
\hline 32 & Universidad Autónoma de Ciudad Juárez & 0 \\
\hline
\end{tabular}


La contribución de las IES en relación con la formación de recursos humanos en programas de posgrado de calidad es otro indicador importante para la identificación de elementos de potencialidad en la producción-comunicación científica, ya que a través de ellos se gesta la formación de nuevos investigadores, tesis y publicaciones que muestren hallazgos de investigaciones recientes.

La regulación de la calidad de los posgrados en México está a cargo del Programa Nacional de Posgrados de Calidad (PNPC), el cual incorpora la generación y aplicación de conocimiento como recurso para el desarrollo de la sociedad, así como la atención de sus necesidades, contribuyendo a consolidar con autonomía y competitividad el crecimiento y desarrollo sustentable del país (PNPC, 2012).
El ingreso de los programas de posgrado en el PNPC, representa un reconocimiento público a su calidad, con base en procesos de evaluación y seguimiento realizados por el comité de pares, por lo que el PNPC coadyuva al Sistema de Garantía de la Calidad de la Educación Superior. Asimismo, es un referente confiable acerca de la calidad de la oferta educativa en el ámbito del posgrado, que ayuda y orienta a los diferentes sectores del país, para que opten por los beneficios que otorga la formación de recursos humanos de alto nivel.

De acuerdo a la tabla 9, el liderazgo en programas pertenecientes al PNPC corresponde a la UnAm que para el 2010 contaba con 134 , en cambio la UACJ y UACH como IES estatales incluidas en esta evaluación, alcanzan 15 y 14 programas reconocidos.

Tabla 9. Total de posgrados en PNPC

\begin{tabular}{|c|l|c|}
\hline Posición respecto al criterio & \multicolumn{1}{|c|}{ IES } & $\begin{array}{c}\text { Total de posgrados } \\
\text { en PNPC }\end{array}$ \\
\hline 1 & Universidad Nacional Autónoma de México & 134 \\
\hline 2 & Universidad de Guadalajara & 66 \\
\hline 3 & Instituto Politécnico Nacional & 61 \\
\hline 4 & Universidad Autónoma de Nuevo León & 58 \\
\hline 5 & Universidad Autónoma Metropolitana & 53 \\
\hline 6 & $\begin{array}{l}\text { Centro de Investigación y de Estudios Avanzados } \\
\text { del IPN }\end{array}$ & 43 \\
\hline 7 & Sistema Tecnológico de Monterrey & 38 \\
\hline 8 & Universidad Autónoma del Estado de México & 37 \\
\hline 9 & Benemérita Universidad Autónoma de Puebla & 36 \\
\hline 20 & Universidad Autónoma de San Luis Potosí & 15 \\
\hline 23 & Universidad Autónoma de Ciudad Juárez & 14 \\
\hline
\end{tabular}


Otros medios de evaluación de la calidad de los programas educativos corresponden a los Comités Interinstitucionales para la Evaluación de la Educación Superior (CIEes), cuyo propósito es contribuir al mejoramiento de la calidad de la educación superior en México, a través de la evaluación diagnóstica de las funciones institucionales de los programas que se ofrecen en las instituciones de ese nivel de estudios; propiciando que los modelos de organización académica y pedagógica orienten al aprendizaje como un proceso a lo largo de la vida, enfocado al análisis, interpretación y buen uso de la información más que a su acumulación. Contribuir a la promoción de la evaluación externa interinstitucional de los programas de docencia, investigación, difusión, administración y gestión de las instituciones de educación superior del país, procurando que los resultados se utilicen en la toma de decisiones de las instituciones educativas y gubernamentales (CieEs, 2012).

De acuerdo a los resultados recolectados de las 10 universidades que mejores indicadores muestran en cuanto al número de programas acreditaros por CieEs (tabla 10), se observa que la Universidad Autónoma del Nuevo León logra el liderazgo, ya que de 307 programas que oferta, 163 están evaluados favorablemente por esta instancia, equivalentes al 53.1\%. En la lista principal aparece una IEs privada (Sistema Tecnológico de Monterrey), correspondiendo además a las IEs locales la posición 19 y 24 de la lista correspondiendo respectivamente a la UACH y a la UACJ.

Tabla 10. Programas acreditador por CIIES

\begin{tabular}{|c|l|c|c|c|}
\hline $\begin{array}{l}\text { Posición } \\
\text { respecto } \\
\text { al criterio }\end{array}$ & \multicolumn{1}{|c|}{ UNIVERSIDAD } & $\begin{array}{c}\text { Total } \\
\text { programas }\end{array}$ & $\begin{array}{c}\text { Programas } \\
\text { en CIES }\end{array}$ & $\begin{array}{c}\text { \% de progra- } \\
\text { mas en CIIES }\end{array}$ \\
\hline 1 & Universidad Autónoma de Nuevo León & 307 & 163 & 53.1 \\
\hline 2 & Universidad de Guadalajara & 399 & 146 & 36.6 \\
\hline 3 & Universidad Veracruzana & 247 & 118 & 47.8 \\
\hline 4 & Universidad Autónoma del Estado de México & 261 & 114 & 43.7 \\
\hline 5 & Benemérita Universidad Autónoma de Puebla & 175 & 100 & 57.1 \\
\hline 6 & Universidad Autónoma de San Luis Potosí & 141 & 70 & 49.6 \\
\hline 7 & Universidad Autónoma de Querétaro & 150 & 68 & 45.3 \\
\hline 8 & Universidad de Colima & 126 & 67 & 53.2 \\
\hline 9 & Universidad Autónoma de Sinaloa & 179 & 63 & 35.2 \\
\hline 10 & Sistema Tecnológico de Monterrey & 1353 & 62 & 4.6 \\
\hline 19 & Universidad Autónoma de Chihuahua & 155 & 48 & 31.0 \\
\hline 24 & Universidad Autónoma de Ciudad Juárez & 93 & 34 & 36.5 \\
\hline
\end{tabular}


Una tercera perspectiva de evaluación de la educación superior y de sus programas educativos en México surge como parte del Programa para la Modernización Educativa 1989-1994 impulsado por el gobierno federal, el cual, desde sus inicios, estableció como acción prioritaria la evaluación interna y externa permanentes de las instituciones para impulsar la mejora de la calidad de los programas educativos y servicios que ofrecen las IEs y como meta la creación de instancias que integraran y articularan un proceso nacional de evaluación de la educación superior, dentro de las cuales se menciona a la Comisión para la Acreditación de la Educación Superior (Copaes), cuya función es regular los pro- cesos de acreditación y dar certeza de la capacidad académica, técnica y operativa de los organismos acreditadores (Copaes, 2012).

De acuerdo a los resultados del análisis, en la tabla 11 se muestra la relación de las 10 IES que poseen mayor número de programas educativos, comparándolos con aquellos que han recibido acreditación por parte de Copaes. La IEs que presenta el mejor indicador es la Universidad Autónoma de Baja California (33.33). Algo interesante en este indicador es que proporcionalmente al número de programas, las instancias estatales tienen un puntaje favorable, ya que la UACJ ocupa la tercera posición $(24.73 \%)$ y la UACH la posición sexta (20.00\%).

Tabla 11. Programas acreditados en COPAES

\begin{tabular}{|c|l|c|c|c|}
\hline $\begin{array}{c}\text { Posición } \\
\text { respecto } \\
\text { al criterio }\end{array}$ & UNIVERSIDADES & $\begin{array}{c}\text { Total de } \\
\text { programas }\end{array}$ & $\begin{array}{c}\text { Programas } \\
\text { acreditados } \\
\text { en COPAES }\end{array}$ & $\begin{array}{c}\text { \% de progra- } \\
\text { mas en COPAES }\end{array}$ \\
\hline 1 & Sistema Tecnológico de Monterrey & 1353 & 187 & 13.82 \\
\hline 2 & Universidad de Guadalajara & 399 & 77 & 19.29 \\
\hline 3 & Universidad Nacional Autónoma de México & 261 & 71 & \\
\hline 27.20 & Universidad Autónoma del Estado de México & 261 & 114 & 43.7 \\
\hline 4 & Instituto Politécnico Nacional & 215 & 52 & 24.18 \\
\hline 5 & Universidad Autónoma de Baja California & 153 & 51 & \\
\hline 33.33 & Universidad Autónoma de Querétaro & 150 & 68 & 45.3 \\
\hline 6 & Universidad Autónoma del Estado de México & 261 & 49 & \\
\hline 18.77 & Universidad Autónoma de Sinaloa & 179 & 63 & 35.2 \\
\hline 7 & Universidad Veracruzana & 247 & 47 & 19.02 \\
\hline 8 & Sistema Universidad del Valle de México & 774 & 41 & \\
\hline 5.29 & Universidad Autónoma de Ciudad Juárez & 93 & 34 & 36.5 \\
\hline 9 & Universidad Autónoma de Sinaloa & 179 & 41 & 22.90 \\
\hline 10 & Universidad Autónoma de Nuevo León & 307 & 40 & 13.02 \\
\hline 16 & Universidad Autónoma de Chihuahua & 155 & 31 & 20.00 \\
\hline 25 & Universidad Autónoma de Ciudad Juárez & 93 & 23 & 24.73 \\
\hline
\end{tabular}


A continuación se desarrolla un análisis más específico sobre las contribuciones científicas del estado de Chihuahua a través de la membrecía en el SNI por IES u organizaciones dedicadas a la investigación.

De acuerdo a los registros del SNI (2012), el Estado de Chihuahua cuenta con 252 miembros pertenecientes a 10 instituciones, tanto de nivel superior, como centros e instituto de investigación y entidades gubernamentales. De la totalidad de los miembros del sNi, el $72.62 \%$ son del género masculino y el $27.38 \%$ del femenino; el $25 \%$ de ellos pertenecen al área de ingenie- rías y $4.37 \%$ al área de medicina y ciencias de la salud, siendo este último el más bajo del total.

Dos IES muestran la mayor aportación: la UACJ y la UACH con 115 y 63 miembros respectivamente, seguidas del Centro de Investigación en Materiales Avanzados (Cimav) con 38, el resto dispersos en cantidades menos significativas en siete instancias (tabla 12). En el caso de la UACH y de la UACJ, su crecimiento entre el 2010 y 2012 fue del 20.63 y 16.52 respectivamente en cuanto al número de miembros en el sNI.

Tabla 12. Relación de miembros del SNI por institución

\begin{tabular}{|c|l|c|c|}
\hline $\begin{array}{c}\text { Posición } \\
\text { respecto } \\
\text { al criterio }\end{array}$ & Institución & $\begin{array}{c}\text { Total de miembros } \\
\text { SNI }\end{array}$ & $\%$ \\
\hline 1 & Universidad Autónoma de Ciudad Juárez & 115 & 45.63 \\
\hline 2 & Universidad Autónoma de Chihuahua & 63 & 25.00 \\
\hline 3 & Centro de Investigación en Materiales Avanzados, S.C. & 38 & 15.08 \\
\hline 4 & Centro de Investigación en Alimentación y Desarrollo, A.C. & 9 & 3.57 \\
\hline 5 & $\begin{array}{l}\text { Dirección General de Educación Superior Tecnológica } \\
\text { (DGEST) }\end{array}$ & 9 & 3.57 \\
\hline 6 & El Colegio de la Frontera Norte, A.C. & 6 & 2.38 \\
\hline 7 & $\begin{array}{l}\text { Instituto Nacional de Investigaciones Forestales y Agrope- } \\
\text { Cuarias }\end{array}$ & 5 & 1.98 \\
\hline 8 & Tecnológico de Monterrey & 3 & 1.19 \\
\hline 9 & Escuela Nacional de Antropología e Historia & 3 & 1.19 \\
\hline 10 & Gobierno del Estado de Chihuahua & 1 & 0.40 \\
\hline Total & 252 & 100 & \\
\hline & & & \\
\hline
\end{tabular}

El nivel de pertenencia al SNI es relevante para la definición de parámetros hacia la producción-comunicación científica, y en mayor medida, cuando se relaciona con el nivel al que pertenece el investigador, ya que de eso depende la exigencia hacia la cantidad/calidad de los productos que de- berá desarrollar. En la tabla 13 se relacionan los datos por institución y sus respectivos niveles, donde se observa que la UACJ y la $\mathrm{UACH}$ muestran liderazgo en cuanto a la cantidad de investigadores reconocidos, aunque un número importante de ellos están ubicados bajo la categoría de candi- 
datos, en cambio en el Cimav predominan las instituciones evaluadas en el estado de los niveles 1, 2 y 3 por encima del resto de Chihuahua.

Tabla 13. Relación de miembros de SNI por nivel

\begin{tabular}{|l|c|c|c|c|}
\hline Organización & \multicolumn{4}{|c|}{ Niveles } \\
\hline & Candidato & $\mathbf{1}$ & $\mathbf{2}$ & $\mathbf{3}$ \\
\hline Universidad Autónoma de Ciudad Juárez & 46 & 61 & 8 & 0 \\
\hline Universidad Autónoma de Chihuahua & 25 & 34 & 4 & 0 \\
\hline $\begin{array}{l}\text { Centro de Investigación en Materiales Avanza- } \\
\text { dos, S.C. }\end{array}$ & 2 & 21 & 9 & 6 \\
\hline $\begin{array}{l}\text { Centro de Investigación en Alimentación y } \\
\text { Desarrollo, A.C. }\end{array}$ & 2 & 5 & 2 & 0 \\
\hline $\begin{array}{l}\text { Dirección General de Educación Superior Tecno- } \\
\text { lógica (DGEST) }\end{array}$ & 1 & 8 & 0 & 0 \\
\hline El Colegio de la Frontera Norte, A.C. & 1 & 3 & 1 & 1 \\
\hline $\begin{array}{l}\text { Instituto Nacional de Investigaciones Forestales } \\
\text { y Agropecuarias }\end{array}$ & 0 & 1 & 0 & 0 \\
\hline Tecnológico de Monterrey & 3 & 0 & 0 & 0 \\
\hline Escuela Nacional de Antropología e Historia & 1 & 1 & 0 & 1 \\
\hline Gobierno del Estado de Chihuahua & 1 & 0 & 0 & 0 \\
\hline Universidad Autónoma de Ciudad Juárez & 2400 & 97 & 5 & 0.002 \\
\hline
\end{tabular}

\section{Conclusiones}

El artículo muestra una serie de indicadores de evaluación relacionados con aspectos que propician o permiten evaluar la capacidad de producción-comunicación científica de las IEs tomando como referencia a actores fundamentales como son los docentes y los programas educativos, así como su proyección en aspectos de reconocimiento por instancias evaluadoras oficiales de alto prestigio, lo cual se debe proyectar en el número y calidad de productos que se generen a través de artículos científicos arbitrados e indizados como referente principal.
Tales indicadores y los resultados observados, a nivel de aproximación, de las 10 IEs mexicanas con mejores promedios, facilitan la posibilidad de derivación de un modelo de evaluación de potencialidades hacia la producción-comunicación científica, tomando como referencia los mejores resultados por IEs en comparación con aquellos datos surgidos de la institución a la cual se pretenda aplicar el modelo, considerando además las proporciones poblacionales que atienden cada una. Deberán considerarse aquellos indicadores que sean comparables entre las IEs participantes ya que no todos aplican a ambas partes. 
Respecto a los resultados del análisis se consideraron 11 indicadores, los cuales se distribuyen de la siguiente forma: 1) matrícula (un indicador), 2) docentes investigadores con reconocimiento (un indicador), 3) producción científica de artículos indizados, arbitrados y de divulgación, y patentes (cuatro indicadores), 4) medios de divulgación de la ciencia (dos indicadores) y 5) evaluación de programas académicos (tres indicadores).

En este estudio, prevalece el liderazgo de la UNAM en nueve de 11 indicadores, se observa que únicamente hay dos indicadores dominados por otras IES (Universidad Autónoma de Nuevo León y Universidad de Baja California).

\section{Referencias}

Braga Ferreira, C., Bernadete Malerbo, M., Silva, M. (2003). Errores en las referencias bibliográficas de la producción académica: un estudio de caso. Scire. Vol. 9. No. 1.

Campos Rosas, J. (2000). La comunicación científica: ¿arte o ciencia? Ars Pharmaceutica, Vol. 41, No. 1.

Codina, L. (2005). SCOPUS: el mayor navegador científico de la web. El profesional de la información, v. 14, n. 1.

Comités Interinstitucionales para la Evaluación de la Educación Superior (2012). Documento electrónico disponible en: http://www.ciees.edu.mx/ciees/quesonciees.php

Consejo Nacional de Ciencia y Tecnología. (2012). Documento electrónico disponible en: www.conacyt. $\mathrm{mx}$.

Consejo para la Acreditación de la Educación Superior, COPAES. (2012). Documento electrónico disponible en: http://www.copaes.org.mx

García-Cepero, M.C. (2010, enero-abril). El estudio de productividad académica de profesores universitarios a través de análisis factorial confirmatorio: el caso de psicología en Estados Unidos de América. Universitas Psychologica, 9, 1: 13-26.

González Nando, E. (2007). La investigación científica en la universidad nacional autónoma de México. Un perfil bibliométricos. México, D.F.: Universidad de Murcia, Facultad de Comunicación y Documentación, Departamento de Información y Comunicación.

Latindex. (2012). Documento electrónico disponible en: www.latindex.unam.mx/

Luna Morales, M., Russell Bernard, J. (2009). El uso de nuevas tecnologías de información y comunicación científica en el área de partículas elementales: el caso de la física mexicana. México, DF. Universidad Nacional Autónoma de México.

Maletta, H. (2009). Epistemología aplicada: metodología y técnica de la producción científica. Lima, Perú: CIES, CEPES, Universidad del Pacífico.

Martínez Escárcega, R., Vega Villarreal, R. (2012, abrilseptiembre). Una mirada a la investigación educativa en Chihuahua a través de los estados de conocimiento. Revista de Investigación Educativa de la REDIECH, año II, no. 4.

Modrego, A., coord. (2002). Capital intelectual y producción científica. Madrid, España: Comunidad de Madrid, Consejería de Educación.

Programa Nacional de Posgrados de Calidad (2012). Documento electrónico disponible en: http://www. conacyt.gob.mx/Becas/calidad/Paginas/Becas_ProgramasPosgradosNacionalesCalidad.aspx

Romanos de Tiratel, S. (2009). Estrategias de difusión del conocimiento en ciencias del hombre: el caso de la Facultad de Filosofía y Letras de la Universidad de Buenos Aires, proyecto UBACYT F157 (Programación científica 2008-2010). Revista Información, Cultura y Sociedad. No. 20, pp. 91-110.

Sistema Nacional de Investigadores. (2012). Documento electrónico disponible en: http://www.conacyt.gob. $\mathrm{mx} /$ sni/Paginas/default.aspx

Universidad Nacional Autónoma de México. Dirección General de Evaluación Institucional. (2010). Estudio comparativo de universidades mexicanas. Documento electrónico disponible en: http://www.ecum. unam.mx/node/1 